\title{
GPS time series modeling by autoregressive moving average method: Application to the crustal deformation in central Japan
}

\author{
Jianxin $\mathrm{Li}^{1}$, Kaoru Miyashita ${ }^{1}$, Teruyuki Kato ${ }^{2}$, and Shinichi Miyazaki ${ }^{3}$ \\ ${ }^{1}$ Department of Environmental Sciences, Ibaraki University, Mito 310-8512, Japan \\ ${ }^{2}$ Earthquake Research Institute, University of Tokyo, Tokyo 113-0032, Japan \\ ${ }^{3}$ Geographical Survey Institute, Tsukuba 305-0811, Japan
}

(Received July 29, 1999; Revised December 8, 1999; Accepted December 24, 1999)

\begin{abstract}
Autoregressive moving average (ARMA) method is applied to modeling the time series of position changes of GPS sites, obtained by the Geographical Survey Institute (GSI) of Japan during the period from April 1996 to March 1998. The present application is focused on denoising of the GPS time series data where only white noise is considered, and detection of data discontinuities and outliers in order to obtain time-averaged velocity and strain fields in central Japan. The data discontinuities are detected by a typical Kalman filter algorithm. The outliers are eliminated by using robust estimation techniques during the ARMA process. The averaged strain field, calculated by the least-squares collocation method from the improved two-year time series data, distinguishes clearly between the tectonically active and inactive regions. Higher maximum shear strain rates were detected in the southern area of the Kanto district. In the areas with very high seismicities, however, the difference between the maximum shear strain rates, that were estimated from the raw time series data and the ARMA-analyzed data, amounted to about 0.2 microstrain/yr. This indicates that the existence of noise and discontinuities can lead to an over-prediction of the strain field.
\end{abstract}

\section{Introduction}

The positions of GPS sites can be determined precisely in recent years. High repeatability of daily positions of the dense GPS network has been attained by the Geographical Survey Institute (GSI) of Japan (Sagiya et al., 1995). At present, more than 940 permanent GPS sites cover the Japanese Islands. The daily position observations of these GPS sites have been used for various studies, such as coseismic movement (Tsuji et al., 1995; Hashimoto et al., 1996) and motion of tectonic plates (Miyazaki and Hatanaka, 1997; Tada et al., 1997). The coseismic crustal deformation associated with the Hokkaido-Toho-Oki earthquake of October 4, 1994 was detected just after the start of regular operations of the GSI's GPS network (Tsuji et al., 1995). Kato et al. (1998) have calculated the strain rates in Japan by using daily coordinate changes of GPS sites.

However, the scatter of the GPS positions is still characterized by errors and seasonal trends (Oware, 1998). Proper techniques should be developed for the automatic identification and calibration of noise and discontinuities in GPS time series. Recently, an error analysis of the GPS daily positions collected at 10 continuously tracking sites in southern California was done with four noise models by Zhang et al. (1997). The result showed that each time series exhibits a linear tectonic signal and a significant colored noise. Discontinuities due to the antenna movements can be corrected by averaging the detrended positions for a few weeks before and

Copy right (C) The Society of Geomagnetism and Earth, Planetary and Space Sciences (SGEPSS); The Seismological Society of Japan; The Volcanological Society of Japan; The Geodetic Society of Japan; The Japanese Society for Planetary Sciences. after the discontinuity and differencing the positions (Bock et al., 1997). This method is not proper to process the large amount of time series data obtained by GSI, which requires that an automatic procedure is used for detecting the discontinuities. In the present research, a method based on the recursive parameter estimation is applied first to detect them. Then the autoregressive moving average (ARMA) method is used to improve the time series of daily position changes. The outliers are eliminated by using robust estimation techniques during the ARMA process. The methods are applied to the practical analysis of GSI's time series data that have been obtained at the sites in central Japan.

\section{Basis of the autoregressive moving average method}

The daily changes in the NS, EW and UP components of the GPS site positions can be extracted from the SINEX data of GSI (Miyazaki and Hatanaka, 1998). In general, we can write the GPS time series as follows:

$$
X(t)=L(t)+S(t)+y(t)
$$

where $X(t)$ corresponds to the daily change in the NS, EW or UP component of the position, $L(t)$ is a linear trend component, $S(t)$ a seasonal component, and $y(t)$ a random noise component. Brockwell and Davis (1991) have summarized some useful techniques for identifying the components in Eq. (1). If we can estimate and extract the deterministic components $L(t)$ and $S(t)$, we can investigate the residual component $y(t)$. After estimating a satisfactory probabilistic model for the process $\{y(t)\}$, we can predict the time series $\{X(t)\}$ along 
with $L(t)$ and $S(t)$. Therefore, the GPS time series analysis actually refers to model the residual component $y(t)$.

The ARMA model can be considered as a special case of the polynomial black-box model, which is frequently used in system identification. Its general form is as follows (Ljung, 1987):

$$
\begin{gathered}
y(t)=H(q, \theta) e(t), \\
H(q, \theta)=\frac{C(q)}{A(q)}, \\
A(q)=1+a_{1} q^{-1}+\ldots+a_{n_{a}} q^{-n_{a}}, \\
C(q)=1+c_{1} q^{-1}+\ldots+c_{n_{c}} q^{-n_{c}}, \\
\theta=\left[\begin{array}{llll}
a_{1} & a_{2} \ldots a_{n_{a}} & c_{1} & c_{2} \ldots c_{n_{c}}
\end{array}\right]^{T},
\end{gathered}
$$

where $y(t)$ corresponds to that in Eq. (1), $q$ is the shift operator, $\theta$ is a vector to describe the time series model, $H(q, \theta)$ is a rational function, $e(t)$ is the white noise with a variance $\sigma$, $A(q)$ and $C(q)$ are polynomials, $n_{a}$ and $n_{c}$ are model orders. $\{y(t)\}$ is assumed to be stationary with even interval. Based on the observations up to time $t-1$, the corresponding onestep-ahead prediction of $y(t)$ can be expressed as follows:

$$
\hat{y}(t \mid \theta)=[1-A(q)] y(t)+[C(q)-1] \varepsilon(t \mid \theta),
$$

where $\varepsilon(t \mid \theta)$ is the prediction error, i.e.,

$$
\varepsilon(t \mid \theta)=y(t)-\hat{y}(t \mid \theta) .
$$

The model parameter $\theta$ can be calculated by minimizing the norm $J_{N}(\theta)$ (Ljung, 1987):

$$
\begin{gathered}
\hat{\theta}(N)=\arg \min _{\theta} J_{N}(\theta), \\
J_{N}(\theta)=\frac{1}{N} \sum_{t=1}^{N} l(t, \theta, \varepsilon(t, \theta)),
\end{gathered}
$$

where arg min means "the minimizing argument of the function", and $l(t, \theta, \varepsilon(t, \theta))$ is a scalar-valued function to measure the prediction error $\varepsilon(t, \theta)$. This way of estimating the model parameter $\theta$ is called the prediction-error identification method (PEM). Further, Ljung (1987) has recommended the damped Gauss-Newton iterative method to calculate the model parameter $\theta$. The quadratic criterion used is as follows:

$$
J_{N}(\theta)=\frac{1}{N} \sum_{t=1}^{N} \frac{1}{2} \varepsilon^{2}(t, \theta) .
$$

It has the gradient

$$
J_{N}^{\prime}(\theta)=-\frac{1}{N} \sum_{t=1}^{N} \psi(t, \theta) \varepsilon(t, \theta),
$$

where $\psi(t, \theta)$ is the gradient matrix of $\hat{y}(t \mid \theta)$ with respect to $\theta$. By applying the Gauss-Newton iterative method, we can obtain

$$
\begin{gathered}
\hat{\theta}_{N}^{(i+1)}=\hat{\theta}_{N}^{(i)}-\mu_{N}^{(i)}\left[J_{N}^{\prime \prime}\left(\hat{\theta}_{N}^{(i)}\right)\right]^{-1} J_{N}^{\prime}\left(\hat{\theta}_{N}^{(i)}\right), \\
J_{N}^{\prime \prime}(\theta)=\frac{1}{N} \sum_{t=1}^{N} \psi(t, \theta) \psi^{T}(t, \theta)-\frac{1}{N} \sum_{t=1}^{N} \psi^{\prime}(t, \theta) \varepsilon(t, \theta),
\end{gathered}
$$

where $\hat{\theta}_{N}^{(i)}$ denotes the $i$-th iterative value. The step size, $\mu_{N}^{(i)}$, is chosen so that

$$
J_{N}\left(\hat{\theta}_{N}^{(i+1)}\right)<J_{N}\left(\hat{\theta}_{N}^{(i)}\right) .
$$

The Akaike information criterion (AIC) (Akaike, 1973) is applied for the selection of the model orders $n_{a}$ and $n_{c}$. After getting the model parameter $\theta$, we can compute the predicted estimates $\hat{y}(t)$.

One important advantage of the ARMA method mentioned above is the detection of outliers in the time series by using robust norms (Ljung, 1987). We define that an observation to be an outlier if $\varepsilon(t, \theta)>2 E\left[\varepsilon^{2}(t, \theta)\right]$. They are very easily detected by plotting the residuals $\varepsilon(t, \theta)$. Figure 1 shows the improved time series at the site of Chichibu by this approach. Observations with red circles are outliers. It can be seen that the noise was decreased greatly, and the changes of the position were clearly shown.

If there are apparent discontinuities in the time series, it might be advisable to analyze the time series by breaking them into homogeneous segments, or by removing the discontinuities. Therefore, it is very important to find the time instants when the abrupt changes occur and to estimate the different models for the different segments during which the system does not change. The algorithm that is implemented in this study is based on the following model description (Ljung, 1987, 1995):

$$
\theta_{0}(t)=\theta_{0}(t-1)+w(t)
$$

where $w(t)$ is zero most of the time, but now and then it abruptly changes the system parameters $\theta_{0}(t) . w(t)$ is assumed to be white Gaussian noise with covariance matrix $R_{1}=E\left[w(t) w^{T}(t)\right]$. For solving this segmentation problem, a typical Kalman filter algorithm (Ljung, 1987) has been given as follows:

$$
\begin{gathered}
\hat{\theta}(t)=\hat{\theta}(t-1)+K(t) \varepsilon(t), \\
\varepsilon(t)=y(t)-\hat{y}(t)=y(t)-\psi^{T}(t) \hat{\theta}(t-1), \\
K(t)=Q(t) \psi(t), \\
Q(t)=\frac{P(t-1)}{R_{2}+\psi^{T}(t) P(t-1) \psi(t)} \\
P(t)=P(t-1)+R_{1}-\frac{P(t-1) \psi(t) \psi^{T}(t) P(t-1)}{R_{2}+\psi^{T}(t) P(t-1) \psi(t)},
\end{gathered}
$$

where $\hat{\theta}(t)$ is the parameter estimate at time $t, \psi(t)$ is the regression vector that contains old values of the observations, $y(t)$ is the observation at time $t$, and $\hat{y}(t)$ is the prediction of the value $y(t)$ from the observations up to time $t-1$ and the current model at time $t-1 . \varepsilon(t)$ is the noise source with the variance, $R_{2}=E\left[\varepsilon^{2}(t)\right]$. The gain $K(t)$ determines how the current prediction error, $y(t)-\hat{y}(t)$, updates the parameter estimate.

The algorithm is specified by $R_{1}, R_{2}, P(0), \theta(0), y(t)$ and $\psi(t)$. Suitable value of $R_{2}$ should be set to evaluate the results, which is the most critical parameter relating to the jump value. In the practical GPS time series analysis, we set the default value of $R_{2}$ to be that it is estimated, i.e., $R_{2}=E\left[\varepsilon^{2}(t)\right] . \quad R_{1}$ is the assumed covariance matrix of 

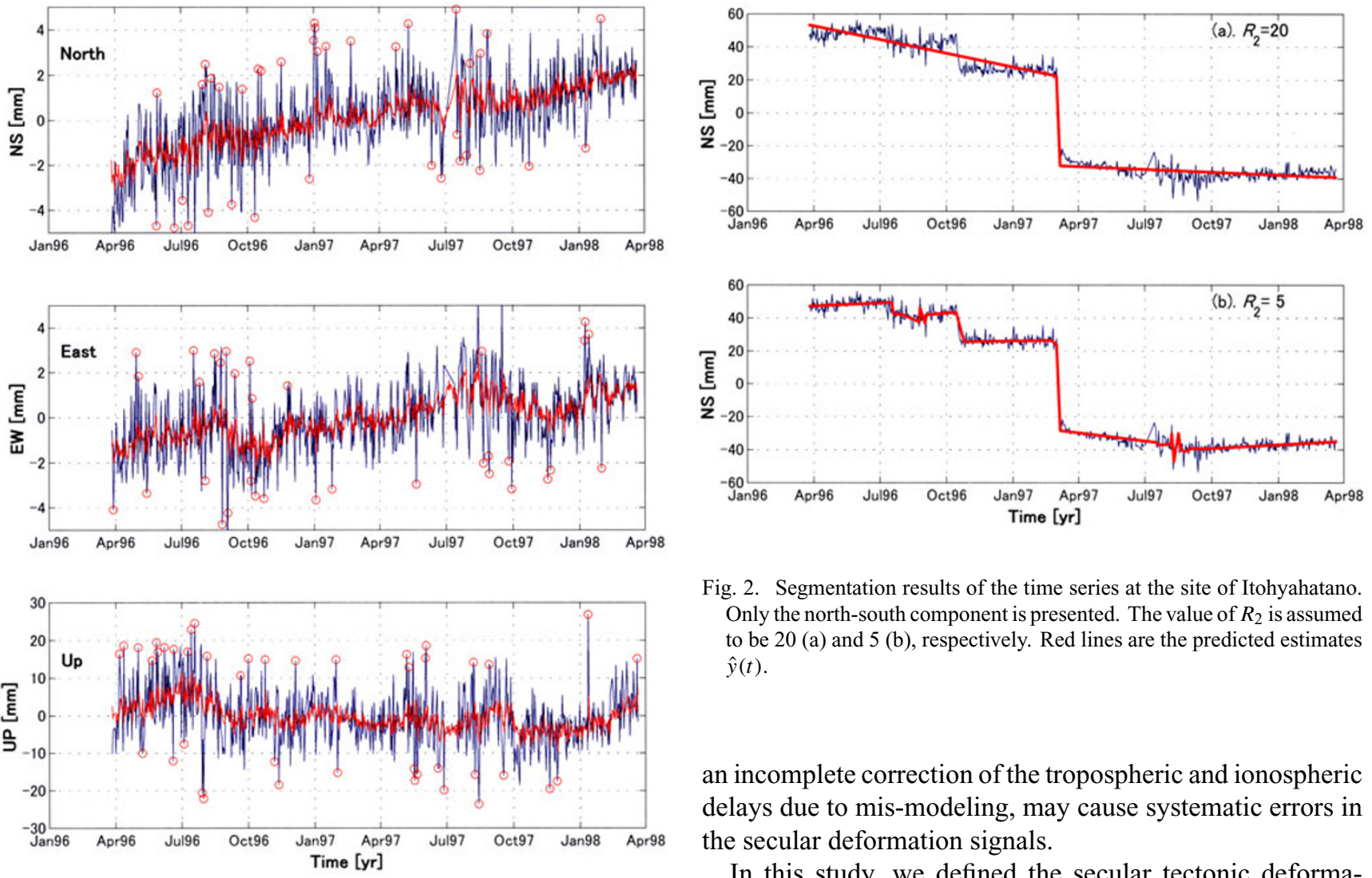

Fig. 2. Segmentation results of the time series at the site of Itohyahatano. Only the north-south component is presented. The value of $R_{2}$ is assumed to be 20 (a) and 5 (b), respectively. Red lines are the predicted estimates $\hat{y}(t)$.

an incomplete correction of the tropospheric and ionospheric delays due to mis-modeling, may cause systematic errors in the secular deformation signals.

In this study, we defined the secular tectonic deformation field as the yearly averaged deformation field without abrupt crustal movements due to earthquakes and/or artificial events. The seasonal changes were removed before applying the ARMA approach by using a polynomial trigonometric fitting method (Li and Tao, 1982).

$$
X(t)=\alpha_{0}+\sum_{j}\left(\alpha_{j} \cos \lambda_{j} t+\beta_{j} \sin \lambda_{j} t\right)+u(t)
$$

the parameter jumps when they occur. Its default value is the identity matrix with the dimension equal to the number of estimated parameters. $\theta(0)$ is the initial value of the parameter, which is set to be zero. While $P(0)$ is the initial covariance matrix of the parameters. Its default is taken to be 10 times the identity matrix. Several Kalman filters are run in parallel to estimate system parameters, each of them corresponding to a particular assumption about when the system actually changes.

Figure 2 shows the result of the segmentation analysis for the site of Itohyahatano. Sudden changes of the position occurred due to the earthquake swarm, which began from March 3, 1997 in and around the Izu peninsula. When $R_{2}$ is set to be a large value, e.g., 20, a big change can be detected out (Fig. 2a). While more complicated segments are detected in the case of a small value of $R_{2}$ (Fig. 2b). However, both of the cases indicate that the time series changed abruptly on March 3 and March 7 when the earthquakes just occurred.

\section{Secular tectonic deformation fields in central Japan}

GPS stations are usually assumed to move linearly or periodically during the observation period. It is important to identify and remove noises and their abrupt changes in order to obtain secular tectonic deformation fields. The causes of the seasonal changes existed in the NS, EW and UP components are still unknown. Rainfall, snow, temperature and/or

where $X(t)$ is the NS, EW or UP component, $u(t)$ is residual, and

$j=1 \sim s / 2$ if $s$ is even;

$j=1 \sim(s-1) / 2$ if $s$ is odd;

$\lambda_{j}=2 \pi j / s$.

$s$ is the number of periods.

The unknown parameters $\alpha_{0}, \alpha_{j}$ and $\beta_{j}$ can be estimated by the least squares method. Moreover, the data discontinuities caused by the earthquakes were also removed by applying the method based on the recursive parameter estimation. The jump values were estimated by forecasting the ARMA processes based on the data before and after the abrupt changes occurred. Though GPS can provide precise three-dimensional positions to suitably equipped users, the vertical components are still determined in the lower precision than the horizontal ones. Thus we focus on the horizontal displacement and strain fields in central Japan.

Figure 3 shows the location of the study area and the complex tectonic geometry of the Eurasian, Okhotsk, Pacific and Philippine Sea plates. The Philippine Sea plate is underthrusting northwestward beneath the Eurasian plate with a convergence rate of 30-40 mm/yr (Seno, 1977; Minster and Jordan, 1979). Several great interplate earthquakes have occurred repeatedly in the region. In the past twenty years, not only conventional geodetic data accumulated in the last 


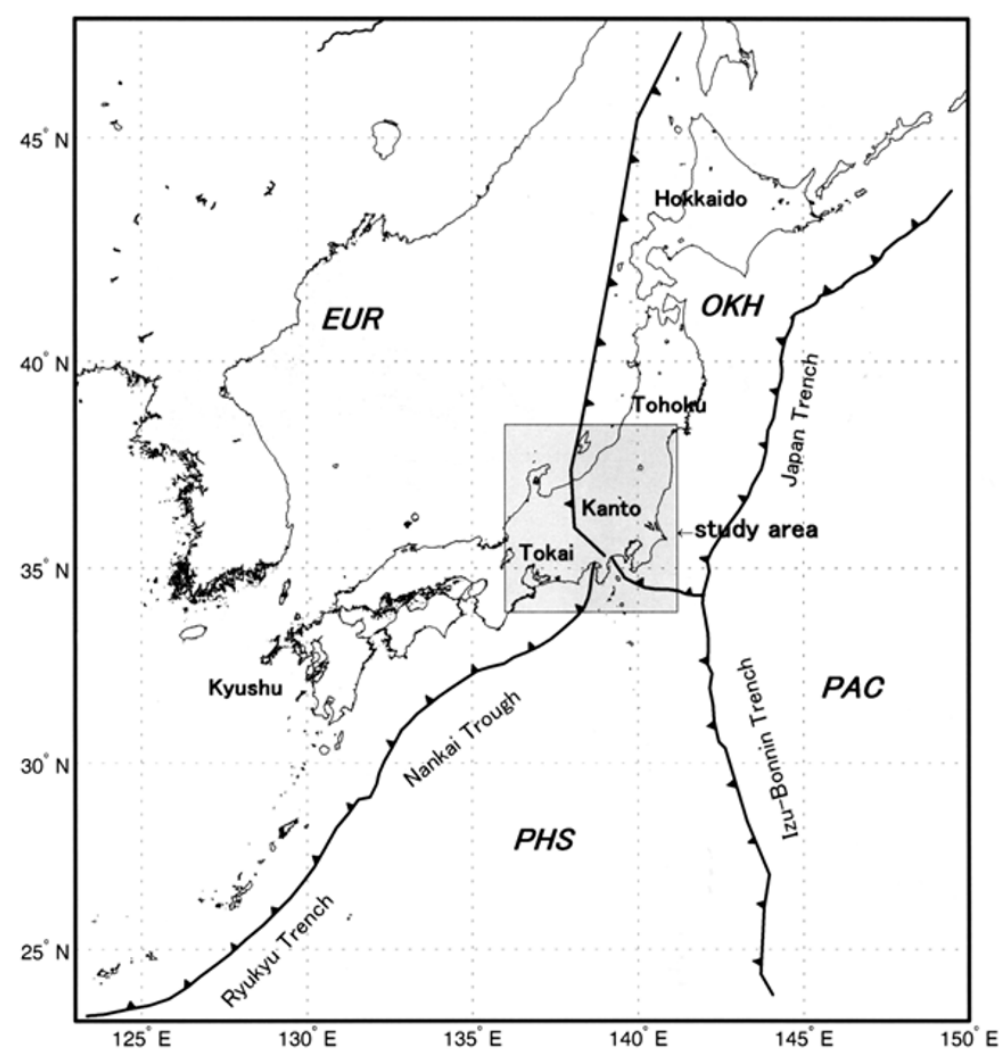

Fig. 3. Location map of the study area (shaded part) and tectonic settings around the Japanese Islands. Thick lines show the plate boundaries. EUR denotes the Eurasian plate; OKH the Okhotsk plate; PAC the Pacific plate; PHS the Philippine Sea plate.

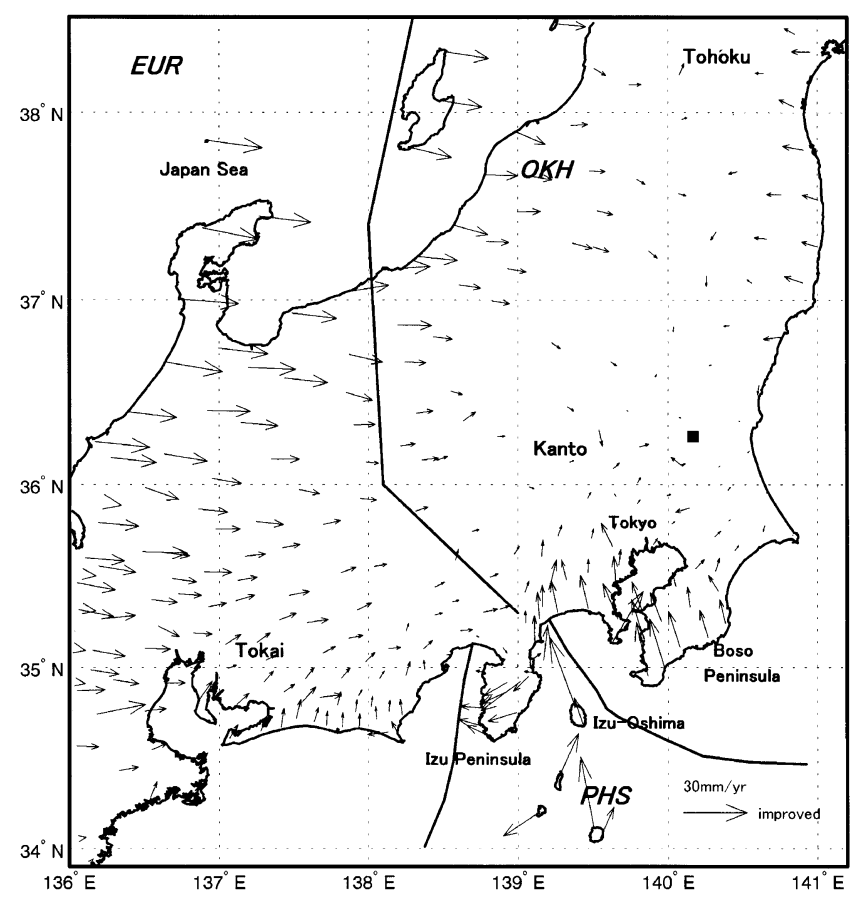

Fig. 4. Two-year averaged velocities at the GPS sites in central Japan, which are estimated relative to the site Yasato. The solid square indicates the fixed site of Yasato.

about 100 years, but also space based geodetic data obtained by VLBI and GPS measurements have been analyzed for crustal deformation and strain fields (Fujii and Nakane, 1983; Shimada and Bock, 1992; Hashimoto and Jackson, 1993).
We can expect that the elimination of noises and detection of discontinuities in the GPS time series data can help interpret the GPS results properly.

\subsection{Yearly averaged velocity field}

The improved time series data indicates that tectonic signals at most of the sites are linear. Therefore, the two-year averaged velocities of the GPS sites relative to the site of Yasato can be estimated by linear regression. As shown in Fig. 4, westward movements along the coast of the Pacific Ocean in the Tohoku district and northward or northwestward movements in the Kanto-Tokai area are prominent. These movements can be considered as the effects of the oceanic plates subducting beneath the Japanese Islands. While eastward movements are dominant on the side of the Japan Sea, which might be understood in terms of the eastward movement of the China continental block due to the northward collision of the India plate (Ishibashi, 1985). In the northern part of the Kanto district, very small displacements are detected out. This result coincides with those obtained by triangulation and trilateration (Fujii et al., 1985). In the area around the Izu peninsula, which is located near the boundary between the Philippine Sea and Eurasian plates, a velocity distribution shows a very complicated pattern. The westward or southwestward movements do not agree with those deduced from the relative plate motion. These anomalous local movements may be caused by the earthquake swarm activities and/or the magma-upwelling activities. On the other hand, Kuwayama and Fujii (1990) have revealed on the basisof the repeated precise levellings that the Izu peninsula 

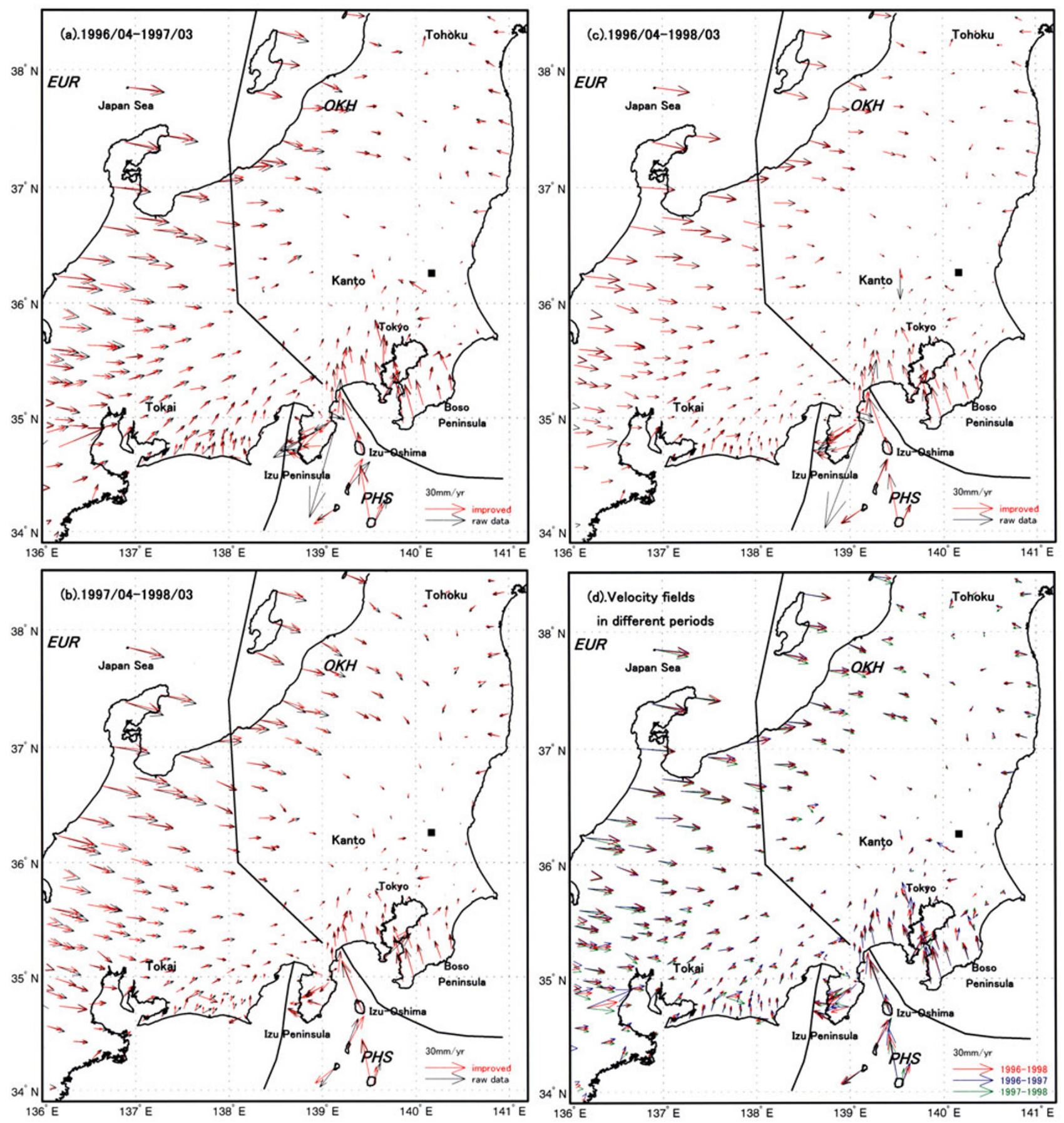

Fig. 5. Comparison between the velocity fields in central Japan, which are estimated from the improved time series data (red lines) and the original ones (black lines) in the different time periods, respectively. (a) is for the time period from April 1996 to March 1997; (b) from April 1997 to March 1998; (c) from April 1996 to March 1998; (d) comparison between the velocity fields deduced from improved data during different time periods.

inclined in the southwestern direction as a whole. The bending of the Philippine Sea plate along almost ENE-WSW direction before subducting beneath the continental plate may cause these anomalous horizontal movements. The NNW movement of the Izu-Oshima Island with a rate of $35 \mathrm{~mm} / \mathrm{yr}$ can be understood in terms of the relative velocity of the Philippine Sea plate against the Eurasian plate. The NNW movements at the southern part of the Boso peninsula can be recognized by the northwestern drag of the Philippine Sea plate against the continental plate (Japanese University Consortium for GPS Research, 1993).
In order to understand the effects before and after applying the ARMA approach, we plotted the velocities in the two shorter periods from April 1996 to March 1997, and from April 1997 to March 1998 (Fig. 5). In the present study area, an earthquake swarm activity began off the eastern coast of the Izu peninsula from March 3, 1997. The significant discrepancies between the velocity fields calculated from the raw time series data and the improved ones are shown at the sites near the earthquake swarm area. Discrepancies in the velocity amounts and directions are also indicated at some of the other sites, especially in the Tokai district (Fig. 5a and 


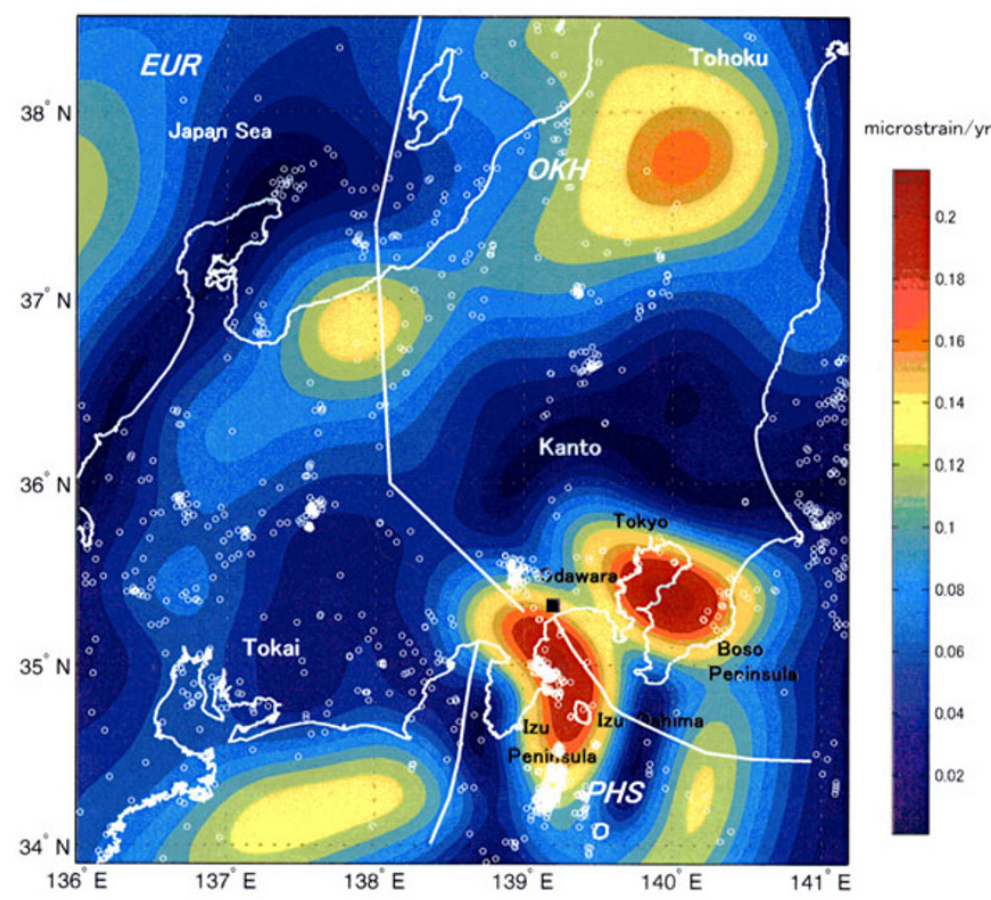

Fig. 6. Maximum shear strain rates in central Japan, estimated from the two-year improved time series data by the least-squares collocation method. White circles indicate epicenters of the earthquakes with depths shallower than $30 \mathrm{~km}$ and magnitudes greater than 3.0 during the period from January 1996 to March 1998, which were determined by the National Research Institute for Earth Science and Disaster Prevention (NIED) of Japan.

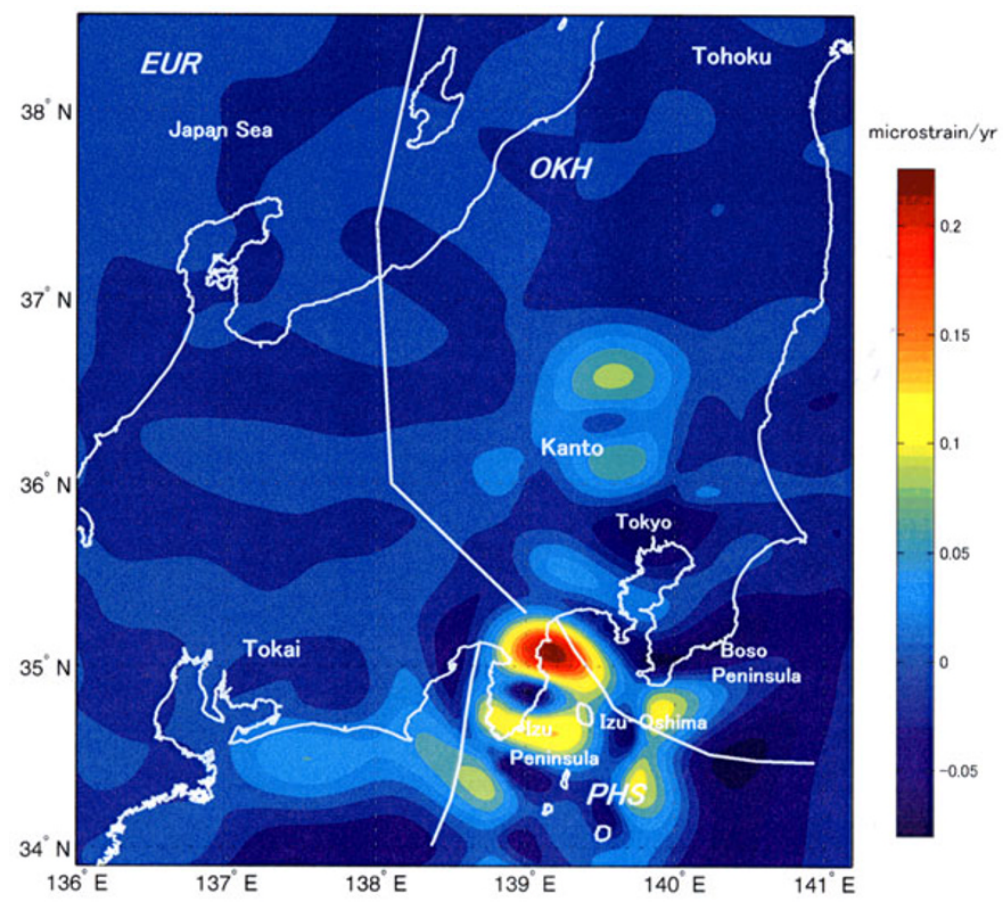

Fig. 7. Discrepancy between the maximum shear strain rates in central Japan estimated from the original time series data and the improved ones. Great differences shown in and around the Izu Peninsula are due to the effect of the data jumps caused by the earthquakes occurred during the observation period. All of these have been removed from the final estimates of the strain rates.

Fig. 5b). For the two-year period from April 1996 to March 1998, the velocity fields coincide with each other, except for the sites at which data discontinuities exist (Fig. 5c). It seems that if data discontinuities and outliers do not exist, long-term time series data can be used to calculate the veloc- ity field directly. Fig. 5d shows a comparison between the velocities deduced from denoised time series data in different periods. It can be seen that velocity vectors agree with each other at most of the GPS sites. This means that the methods mentioned above are effective for the short time series. 


\subsection{Yearly averaged maximum shear strain field}

The goal of this study is to estimate the secular strain field in central Japan by using the improved time series data. The strain components were then computed by the least-squares prediction method which includes parameter estimation, filtering and prediction by least-squares adjustment (e.g., Hein and Kistermann, 1981; El-Fiky et al., 1997; Kato et al., 1998). The velocities at uniform $7 \mathrm{~km} \times 7 \mathrm{~km}$ grids were interpolated by adopting the empirical covariance function and then differentiated with respect to space to estimate strain rates (Kato et al., 1998). Figure 6 shows the estimated maximum shear strain rates in central Japan. The white circles indicate epicenters of the shallow earthquakes from January 1996 to March 1998, which have been determined by the National Research Institute for Earth Science and Disaster Prevention (NIED) of Japan. Higher maximum shear strain rates of about $0.23 \mathrm{microstrain} / \mathrm{yr}$ are shown in and around the Izu and Boso peninsulas. The area of the higher maximum shear strain rates in the eastern Izu peninsula includes the earthquake swarm areas. The axis of the peak contour changes from the N-S direction in the southern part to the NW-SE direction in the northern part and extends to Odawara, which has been designated as an intensified observation area. A disastrous earthquake has been expected to occur around the turn of the next century (Ishibashi, 1985). Relatively high maximum shear strain rates are also shown in the southern Tohoku district, although few shallow earthquakes occurred in this region. Another notable area with relatively high maximum shear strain rates is located along the coast of the Japan Sea, which nearly corresponds to the Shinanogawa seismic zone. It seems that the shallow earthquakes have generally occurred in and around the areas with higher strain rates. On the other hand, the areas with lower strain rates can be found in the northern Kanto and northern Tokai districts. These agree with the results estimated from the last 100-year triangulation and trilateration to some extent (Hashimoto, 1990). Hashimoto (1990) explained that compressional forces due to the subduction of the Philippine Sea plate may not be transmitted to the northern Kanto district. All of these results from the GPS measurements may be meaningful for the earthquake prediction in the future.

The effects of the noises, especially the data discontinuities, on the velocity field have been demonstrated in Fig. 5 . Their effects on the short-term time series data are very notable, and evident in the strain field in Fig. 7. The differences between the results from the raw and improved data generally become larger in the areas where the positions of the GPS sites change abruptly due to earthquakes during the observation period. The maximum discrepancy amounts to about $0.22 \mathrm{microstrain} / \mathrm{yr}$ in and around the Izu peninsula and the southern part of the Boso peninsula. This means that we should carefully remove data discontinuities and/or outliers, and denoise the GPS time series data before estimating the secular strain.

\section{Conclusion}

We applied the ARMA method to analyze the GPS time series data obtained during the period from April 1996 to March 1998. The utility of time series data is improved greatly when outliers, seasonal changes and data disconti- nuities are removed. Such an analysis is especially necessary when the observation period is short. We demonstrated some important results concerning the secular tectonic deformation fields, i.e., yearly averaged velocities and yearly averaged maximum shear strain rates in central Japan. The tectonically active and inactive regions could be clearly distinguished. Higher maximum shear strain rates were detected out in the eastern part of the Izu peninsula, the southern part of the Kanto district and the area along the coast of the Japan Sea. We also discussed the effects of the noises of the time series data on the results of the maximum shear strain rate. The discrepancy between the results from the raw and improved time series data amounted to about 0.22 microstrain/yr, implying that discontinuities can lead to an over-prediction of the secular strain field.

Acknowledgments. The authors are indebted to Y. Fujii for his valuable suggestions. We extend our thanks to G. S. El-Fiky and E. $\mathrm{N}$. Oware for the use of their program for computing crustal strain and helpful discussions. The comments and careful reviews from reviewers, K. Heki, A. Mao and F. Webb, improved the manuscript greatly.

\section{References}

Akaike, H., Information theory and an extension of the maximum likelihood principle, in 2nd International Symposium on Information Theory, edited by B. N. Petrov and F. Csaki, pp. 267-281, Akademiai Kiado, Budapest, 1973.

Bock, Y., S. Wdowinski, P. Fang, J. Zhang, S. Williams, H. Johnson, J. Behr, J. Genrich, J. Dean, M. van Domselaar, D. Agnew, F. Wyatt, K. Stark, B. Oral, K. Hudnut, R. King, T. Herring, S. Dinardo, W. Young, D. Jackson, and W. Gurtner, Southern California Permanent GPS Geodetic Array: Continuous measurements of regional crustal deformation between the 1992 Landers and 1994 Northridge earthquakes, J. Geophys. Res., 102, 18013-18033, 1997.

Brockwell, P. J. and R. A. Davis, Time Series: Theory and Methods, 577 pp., Springer-Verlag New York, Inc., 1991.

El-Fiky, G. S., T. Kato, and Y. Fujii, Distribution of vertical crustal movement rates in the Tohoku district, Japan, predicted by least-squares collocation, J. Geod., 71, 432-442, 1997.

Fujii, Y. and N. Nakane, Horizontal Crustal Movements in the Kanto-Tokai District, Japan, as Deduced from Geodetic Data, Tectonophys., 97, 115 140, 1983.

Fujii, Y., K. Miyashita, and Y. Kitazawa, Crustal deformation and seismicity in and around the Ibaraki prefecture, Earth Mon., 7, 2, 79-84, 1985 (in Japanese).

Hashimoto, M., Horizontal strain rates in the Japanese islands during interseismic period deduced from geodetic surveys (Part I): Honshu, Shikoku and Kyushu, Zisin, 43, 13-26, 1990 (in Japanese with English abstract).

Hashimoto, M. and D. Jackson, Plate Tectonics and Crustal Deformation Around the Japanese Islands, J. Geophys. Res., 98, 16149-16166, 1993.

Hashimoto, M., T. Sagiya, H. Tsuji, Y. Hatanaka, and T. Tada, Co-seismic displacements of the 1995 Hyogo-ken Nanbu Earthquake, J. Phys. Earth, 44, 255-279, 1996.

Hein, G. A. and R. Kistermann, Mathematical foundation of nontectonic effects in geodetic recent crustal movement models, Tectonophys., $\mathbf{7 1}$ 315-334, 1981.

Ishibashi, K., On the mechanism of the Tokai earthquake, Earth Mon., 7, 128-131, 1985 (in Japanese).

Ishibashi, K., A possible large earthquake near Odawara city in near future, Earth Mon., 7, 420-426, 1985 (in Japanese).

Japanese University Consortium for GPS Research, Crustal Deformation Observed at the Sagami Bay Area, Japan, by Means of GPS Interferometry, J. Geod. Soc. Japan, 39, 107-119, 1993.

Kato, T., G. S. El-Fiky, E. N. Oware, and S. Miyazaki, Crustal strains in the Japanese islands as deduced from dense GPS array, Geophys. Res. Lett., 25, 3445-3448, 1998.

Kuwayama, T. and Y. Fujii, Change of mode of crustal deformation in the Izu peninsula, Honshu, Japan and bending of the Northernmost part of the Philippine Sea plate, Zisin, 43, 101-110, 1990 (in Japanese with English 
abstract).

Li, Q. and B. Tao, Theory of Probability and Statistics, and Its Applications to Geodesy, 321pp., Survey and Mapping, China, 1982 (in Chinese).

Ljung, L., System identification, theory for the user, 519 pp., Prentice-Hall, New York, 1987.

Ljung, L., System identification toolbox for use with Matlab-user's guide, Natick, Mass., 266 pp., The MathWorks, Inc., 1995.

Minster, J. B. and Y. H. Jordan, Rotation vectors for the Philippine Sea and Vivera Plates, Eos Trans. AGU, 60, 958, 1979.

Miyazaki, S. and Y. Hatanaka, Crustal deformation observed by GSI's new GPS array, Eos Trans. AGU, 78, S104, 1997.

Miyazaki, S. and Y. Hatanaka, About Continuous GPS monitoring system of GSI, in Kishyo Kenkyu Note, edited by H. Nakamura, 192, 105-131, Meteorological Society of Japan, 1998 (in Japanese).

Oware, E. N., A New Tectonic View of the Japanese Islands Based on GPS Dense Array Data, Ph.D. thesis, 214 pp., University of Tokyo, 1998.

Sagiya, T., A. Yoshimura, E. Iwata, K. Abe, I. Kimura, K. Uemura, and T. Tada, Establishment of Permanent GPS Observation Network and Crustal Deformation Monitoring in the Southern Kanto and Tokai Areas, Bull. Geogr. Surv. Inst., 41, 105-118, 1995.
Seno, T., The Instantaneous Rotation Vector of the Philippine Sea Plate Relative to the Eurasian Plate, Tectonophys., 42, 209-226, 1977.

Shimada, S. and Y. Bock, Crustal Deformation Measurements in Central Japan Determined by a Global Positioning System Fixed-Point Network, J. Geophys. Res., 97, 12437-12455, 1992.

Tada, T., T. Sagiya, and S. Miyazaki, Deforming Japanese islands as seen from GPS observations, Kagaku, 67, 917-927, 1997 (in Japanese).

Tsuji, H., Y. Hatanaka, T. Sagiya, and M. Hashimoto, Coseismic crustal deformation from the 1994 Hokkaido-Toho-Oki Earthquake Monitored by a nationwide continuous GPS array in Japan, Geophys. Res. Lett., 22, 1669-1672, 1995.

Zhang, J., Y. Bock, H. Johnson, P. Fang, S. Williams, J. Genrich, S. Wdowinski, and J. Behr, Southern California Permanent GPS Geodetic Array: Error analysis of daily position estimates and site velocities, $J$. Geophys. Res., 102, 18035-18055, 1997.

J.Li(e-mail: ljx@mito.ipc.ibaraki.ac.jp), K. Miyashita(e-mail: miyas@) mito.ipc.ibaraki.ac.jp), T. Kato, and S. Miyazaki 